\title{
Caracterização antropométrica de portadores de câncer de próstata do Ceará, Brasil 1
}

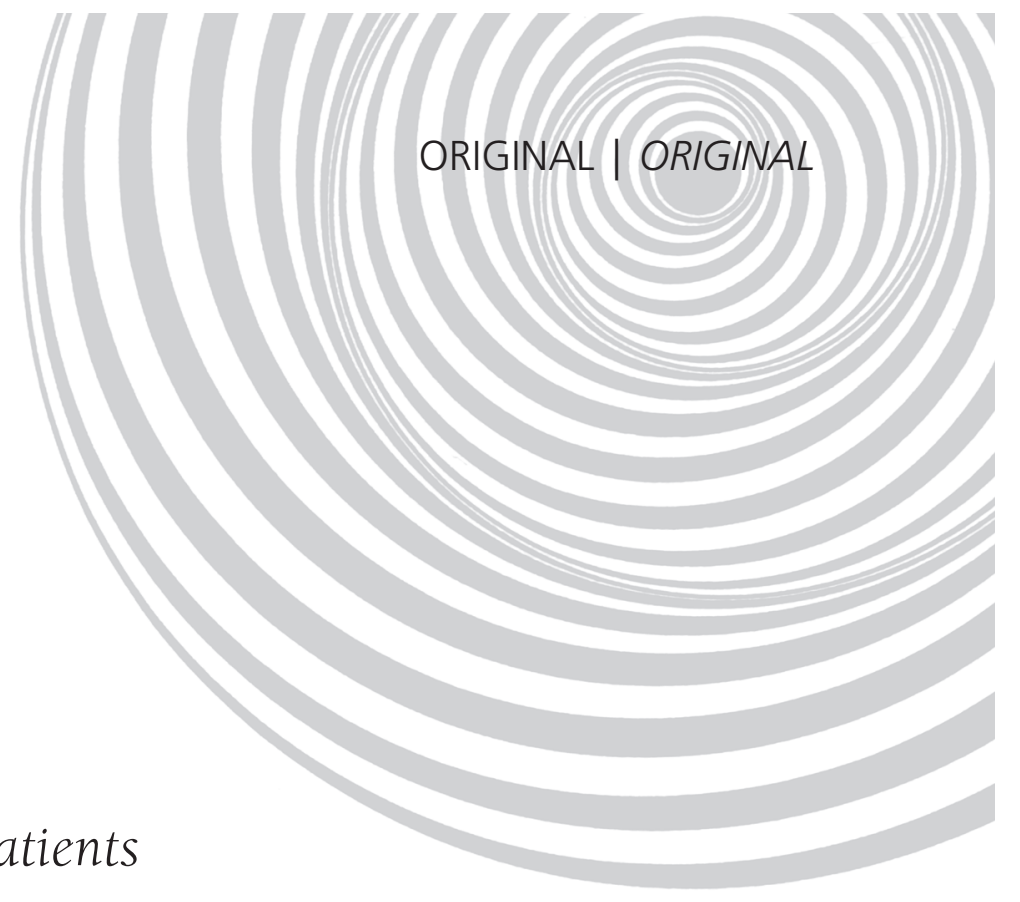

\section{Anthropometric characterization of patients with prostate cancer in Ceará, Brazil}

Soraia Pinheiro MACHADO²

Helena Alves de Carvalho SAMPAIO ${ }^{3}$

José Wellington de Oliveira LIMA4

\section{RE S U M O}

\section{Objetivo}

Este estudo teve como objetivo determinar a situação antropométrica de portadores de câncer de próstata, com foco específico na determinação da proporção de excesso de peso e obesidade e do grau de risco para desenvolver doenças crônicas.

\section{Métodos}

Trata-se de um estudo descritivo, englobando 220 homens com diagnóstico de câncer de próstata, atendidos em uma instituição de referência do Ceará, entre março e agosto de 2006. Os pacientes responderam a um formulário semi-estruturado contendo dados demográficos e socioeconômicos e peso habitual anterior ao diagnóstico da doença. Tiveram aferidas as medidas antropométricas de peso, altura e circunferência da cintura. Calculou-se o índice de massa corporal, tanto atual como habitual, para classificação do estado nutricional, segundo parâmetros da Organização Mundial de Saúde; também a circunferência da cintura foi avaliada segundo os parâmetros da mesma entidade. Compararam-se as distribuições pareadas, anterior e atual, do peso e do Índice de Massa Corporal pelo teste dos postos sinalizados de Wilcoxon.

\section{Resultados}

A proporção atual de excesso de peso e obesidade nos pacientes estudados foi, respectivamente, de 53,64\% e $15,0 \%$, e de $54,23 \%$ e $15,92 \%$, no passado, sem diferença estatisticamente significante com o passar do tempo $(p=0,710)$. A maioria deles $(61,28 \%)$ apresentou risco aumentado ou alto risco para desenvolver doenças crônicas.

\footnotetext{
1 Artigo elaborado a partir da dissertação de S.P. MACHADO, intitulada "Fatores nutricionais associados ao câncer de próstata na cidade de Fortaleza, Ceará". Universidade Estadual do Ceará; 2007.

2 Universidade Federal do Maranhão, Curso de Nutrição, Departamento de Ciências Fisiológicas. Av. dos Portugueses, s/n., Campus Universitário do Bacanga, Prédio 3, 65080-040, São Luís, MA, Brasil. Correspondência para/Correspondence to: S.P. MACHADO.E-mail:<soraiamachado@yahoo.com.br>.

3 Universidade Estadual do Ceará, Curso de Nutrição, Curso de Mestrado Acadêmico em Saúde Pública. Fortaleza, CE, Brasil.

${ }^{4}$ Universidade Estadual do Ceará, Curso de Medicina. Fortaleza, CE, Brasil.
} 
368 | S.P. MACHADO et al.

\section{Conclusão}

A alta prevalência, passada e atual, de excesso de peso e obesidade, associada à presença de gordura abdominal verificada no grupo, representa risco para o desenvolvimento de doenças crônicas em geral.

Termos de indexação: Antropometria. Neoplasias da próstata. Obesidade. Peso corporal.

\section{A B S T R A C T}

\section{Objective}

The objective of this study was to determine the anthropometric status of patients with prostate cancer, focusing specifically on the determination of the proportion of excess weight and obesity and the risk of developing chronic diseases.

\section{Methods}

This descriptive study included 220 men with a diagnosis of prostate cancer attending a referral center in Ceará, Brazil, from March to August 2006. A semi-structured form was used to obtain the patients' demographic and socioeconomic data and average weight before diagnosis. Height, weight and waist circumference were measured. Current and previous body mass indices were calculated to determine nutritional status according to the World Health Organization's criteria. Waist circumference was also classified according to the WHO's criteria. The two-sample paired Wilcoxon signed-rank test was used to compare the distributions of previous and current weight and body mass index.

\section{Results}

The current proportion of excess weight and obesity in the studied patients were $53.64 \%$ and $15.0 \%$ respectively and the previous prevalences were $54.23 \%$ and $15.92 \%$ respectively. There was no significant statistical difference over time $(p=0.710)$. Most patients (61.28\%) were at high risk of developing chronic diseases.

\section{Conclusion}

The high current and previous prevalences of excess weight and obesity associated with abdominal obesity are risk factors for chronic diseases.

Indexing terms: Anthropometry. Prostatic neoplasm. Obesity. Body Weight.

\section{N T R O D U Ç Ã O}

O câncer tem aparecido como um importante problema de saúde pública em todo o mundo, especialmente nos países em desenvolvimento, devido ao acelerado crescimento da população de faixa etária mais elevada. A World Health Organization e a Food and Agriculture Organization (WHO/FAO) estimam que as taxas globais de câncer possam crescer $50 \%$ entre os anos de 2000 e 2020, passando de 10 para 15 milhões de novos casos da doença. A enfermidade representa a segunda causa de mortes no mundo, sendo geralmente superada apenas pelas doenças cardiovasculares ${ }^{1}$.

Outro problema que vem ganhando cada vez maior destaque no cenário mundial da saúde pública é o excesso de peso, devido ao rápido aumento de sua prevalência em todo o mundo e especificamente no Brasil, onde antes era um problema bem menos evidenciado². Dados estimados da Organização Mundial de Saúde apontaram, no ano de 2002, a existência de mais de um bilhão de adultos com excesso de peso, sendo 300 milhões considerados obesos ${ }^{1}$. Dados nacionais da Pesquisa de Orçamentos Familiares (POF) 2002-2003 mostram que, entre os homens brasileiros com mais de 20 anos, houve um crescimento de peso excedente (Índice de Massa Corporal - IMC $\geq 25 \mathrm{~kg} / \mathrm{m}^{2}$ ) entre os anos de 1974 e 2003, passando a prevalência de 18,6\% para $41,0 \%$. Para a obesidade (IMC $\left.\geq 30 \mathrm{~kg} / \mathrm{m}^{2}\right)$, esse percentual elevou-se de 2,8\% para 8,8\% no mesmo período. Regionalmente, 32,9\% dos homens 
nordestinos apresentam excesso de peso e 6,7\% são obesos. Entre os cearenses, estes valores são, respectivamente, $35,1 \%$ e $7,9 \%^{2}$.

O sobrepeso e a obesidade contribuem fortemente para a carga de doenças crônicas e de incapacidades, que vão desde dificuldades respiratórias até condições graves, como doença coronariana e certos tipos de câncer ${ }^{3}$. Estima-se que 90 mil mortes por câncer poderiam ser evitadas a cada ano se a população adulta mantivesse um peso corporal adequado ${ }^{4}$ e que, aproximadamente, $20-33 \%$ dos casos de câncer mais comuns possam ser atribuídos ao peso corporal excedente e à inatividade física'.

Embora muitos estudos tenham demonstrado que o excesso de peso aumenta o risco de câncer do cólon, rim, esôfago, endométrio e de mama (este em mulheres pós-menopausa), dando suporte à idéia de que o controle da obesidade pode representar uma importante medida de prevenção de muitos tipos de câncer na sociedade moderna ${ }^{5}$, especificamente para o câncer da próstata, os efeitos do excesso de peso em sua gênese ainda são controversos ${ }^{5-7}$, sendo os resultados dos estudos nessa área inconsistentes e contraditórios ${ }^{8-10}$.

Assim sendo e, considerando o aumento da mortalidade por câncer de próstata que vem ocorrendo paralelamente à elevação da prevalência de sobrepeso e obesidade no Brasil e em todo o mundo, torna-se relevante a realização deste estudo, que teve como objetivo determinar a situação antropométrica de portadores de câncer de próstata, com foco específico na determinação da proporção de excesso de peso e obesidade, bem como do grau de risco para o desenvolvimento de doenças crônicas, dada a maior controvérsia existente quanto à inter-relação destes indicadores com a doença, como já referido. Outro aspecto positivo deste estudo é a investigação de um tema pouco explorado em trabalhos nacionais.

\section{M É T O D O S}

Este estudo integra o trabalho de dissertação de mestrado intitulada "Fatores nutricionais associados ao câncer de próstata na cidade de Fortaleza, Ceará", que foi apresentada ao Curso de Mestrado Acadêmico de Saúde Pública (CMASP) da Universidade Estadual do Ceará (UECE), com apoio do Conselho Nacional de Desenvolvimento Científico e Tecnológico (CNPq), e que foi devidamente submetido e aprovado pelo Comitê de Ética em Pesquisa da UECE, de acordo com o protocolo de n 05464391-0.

Trata-se de um estudo descritivo, com base em dados obtidos a partir de entrevistas com os sujeitos da pesquisa e da aferição de medidas antropométricas, cuja coleta ocorreu durante o período de março a abril de 2006 . A população do estudo foi constituída de homens com diagnóstico prévio de câncer de próstata, clientes do Serviço de Uro-Oncologia do Instituto do Câncer do Ceará (ICC), instituição de referência estadual para prevenção e tratamento da doença. A amostra compreendeu 220 pacientes, correspondente ao total de indivíduos atendidos no período citado no referido Serviço, e que preencheram os seguintes critérios de inclusão: residir no estado do Ceará há, pelo menos, 15 anos e concordar em participar do estudo mediante a assinatura do Termo de Consentimento Livre e Esclarecido.

Todos responderam a um questionário semi-estruturado, aplicado na própria Instituição, contendo os seguintes dados: demográficos e socioeconômicos (nome, idade, escolaridade e renda familiar mensal) e peso habitual anterior ao diagnóstico da doença. Tiveram aferidas, segundo técnicas padronizadas pela World Health Organization (WHO), as medidas antropométricas de peso, altura e circunferência da cintura (CC $)^{11}$. Para tanto, foram utilizadas balança antropométrica da marca Filizola ${ }^{\circledR}$, com capacidade de $150 \mathrm{~kg}$ e intervalo de $100 \mathrm{~g}$, cujo antropômetro acoplado possui capacidade de $2 \mathrm{~m}$ e intervalo de $0,5 \mathrm{~cm}$, além de trena métrica inelástica flexível.

Calculou-se o Índice de Massa Corporal (peso/altura ${ }^{2}$ ) para classificação do estado nutricional, segundo a $\mathrm{WHO}^{11}$, e aferiu-se a circunferência da cintura, para determinar o tipo de distribuição de gordura corpórea, estimando-se o grau 
de risco para o desenvolvimento de doenças crônicas, considerando risco diminuído $C C<94 \mathrm{~cm}$, risco aumentado $C C \geq 94$ e $<102 \mathrm{~cm}$ e alto risco $C C \geq 102 \mathrm{~cm}^{11}$.

O estado nutricional do grupo foi avaliado tanto com base no IMC atual, utilizando-se o peso aferido no momento da entrevista, como no IMC habitual, usando-se o peso referido pelo entrevistado como aquele observado no período anterior à descoberta da doença e ao início do tratamento. Os critérios de classificação utilizados foram também da $\mathrm{WHO}^{11}$, sendo considerado excesso de peso, $I M C \geq 25 \mathrm{~kg} / \mathrm{m}^{2}$ e obesidade, $I M C \geq 30 \mathrm{~kg} / \mathrm{m}^{2}$. A evolução do peso corporal nos últimos anos foi calculada pela diferença entre o peso atual e o peso habitual.

Os dados foram tabulados para apresentação em freqüência simples e percentual, médias e variações. A análise dos dados deu-se de forma descritiva, além de comparar as distribuições pareadas, antes e após o diagnóstico da doença e o início do tratamento, do peso e do IMC por meio do Teste dos Postos Sinalizados de Wilcoxon, tendo sido consideradas significantemente diferentes as distribuições cuja probabilidade de serem semelhantes foi menor que 0,05 (valor- $p<0,05)$. A escolha deste teste deveu-se às distribuições do peso anterior e atual e do IMC anterior e atual, que se apresentarem significantemente diferentes de uma distribuição normal (valor- $p<0,05$ a um Teste de Assimetria e Curtose para Normalidade).

\section{RES ULTADOS}

Os portadores de câncer de próstata estudados apresentaram uma idade média de 72,88 anos, variando de 52 a 97 anos.

A maioria dos pacientes $(55,0 \%)$ vinha do interior do estado, sendo 32 (14,5\%) da região serrana, $19(8,6 \%)$ de cidades praianas e 70 $(31,8 \%)$ do sertão cearense. O restante (45\%) era proveniente da capital do estado (Fortaleza).

Em relação à escolaridade, a grande maioria $(82,3 \%)$ não chegou sequer a concluir o ensino fundamental, destacando-se, ainda, que $88(40,0 \%)$ nunca freqüentaram a escola. Quanto à renda, observou-se que a maior parte da amostra $(81,4 \%)$ informou uma renda familiar mensal inferior a cinco salários-mínimos (SM). A renda familiar mensal média encontrada no grupo foi de 4,02 salários-mínimos, com uma extensa variação $(0,57-45,71 \mathrm{SM})$.

A Tabela 1 traz os dados relativos ao perfil antropométrico do grupo estudado. O peso atual do grupo, aferido no dia da entrevista, variou de 34 a 99,7kg, com uma média de 67,64kg. Valores semelhantes (Tabela 2) foram observados com relação ao peso habitual antes da doença e do início do tratamento, relatado pelos entrevistados, para o qual se verificou um peso médio de $68,2 \mathrm{~kg}$ (45-97kg). Dezenove pacientes (8,6\%) não souberam informar seu peso habitual.

Com relação à estatura do grupo estudado, obteve-se um valor médio de $162,21 \mathrm{~cm}$, variando de 146,7 a $181,6 \mathrm{~cm}$.

No que se refere à evolução do peso corporal dos portadores de câncer de próstata, observou-se que, dos 201 que souberam informar o peso habitual para tal comparação, 192 (95,5\%) sofreram alguma alteração de peso, tendo sido a média de alteração igual a 5,1 kg (0,10-22,00kg). A alteração do peso corporal foi superior a $5 \mathrm{~kg}$ em 36,8\% desses homens. Predominou a perda de peso $(49,3 \%)$, embora com variações levemente menores que com relação ao ganho de peso $(46,3 \%)$. Os valores médios de variação de peso foram $5,1 \mathrm{~kg}(0,10-22,00 \mathrm{~kg})$ e $5,5 \mathrm{~kg}$ $(0,10-19,8 \mathrm{~kg})$, para o grupo que perdeu peso e para o grupo que ganhou peso, respectivamente.

Quanto ao estado nutricional, observou-se um leve predomínio de excesso de peso (53,6\%), com base no IMC atual, sendo que em nenhum dos pacientes constatou-se obesidade de grau III. O IMC atual médio encontrado foi de $25,71 \mathrm{~kg} / \mathrm{m}^{2}$ $\left(12,80-38,9 \mathrm{~kg} / \mathrm{m}^{2}\right)$. Quando avaliados, de acordo com o IMC calculado com o referido peso habitual anterior à doença, os valores foram semelhantes (Tabela 2), verificando-se um excesso de peso em 
Tabela 1. Características antropométricas de portadores de câncer de próstata. Fortaleza (CE), 2006.

\begin{tabular}{|c|c|c|c|c|}
\hline \multirow{2}{*}{ Classificação } & \multicolumn{2}{|c|}{ Atual } & \multicolumn{2}{|c|}{ Anterior à doença } \\
\hline & $n$ & $\%$ & $n$ & $\%$ \\
\hline \multicolumn{5}{|c|}{ Peso corporal (kg) } \\
\hline$<60$ & 55 & 25,0 & 54 & 26,9 \\
\hline $60-67$ & 50 & 22,7 & 48 & 23,9 \\
\hline $68-75$ & 62 & 28,2 & 54 & 26,9 \\
\hline$>75$ & 53 & 24,1 & 45 & 22,4 \\
\hline Total & 220 & 100,0 & 201 & 100,0 \\
\hline \multicolumn{5}{|c|}{ Altura $(\mathrm{cm})$} \\
\hline$\leq 154$ & 25 & 11,4 & - & - \\
\hline $154-163$ & 94 & 42,7 & - & - \\
\hline $163-172$ & 91 & 41,4 & - & - \\
\hline$>172$ & 10 & 4,5 & - & - \\
\hline Total & 220 & 100,0 & - & - \\
\hline \multicolumn{5}{|c|}{ Índice de Massa Corporal } \\
\hline Desnutrição & 4 & 1,8 & 2 & 1,0 \\
\hline Normalidade/eutrofia & 98 & 44,5 & 90 & 44,8 \\
\hline Sobrepeso & 85 & 38,6 & 77 & 38,3 \\
\hline Obesidade grau I & 29 & 13,2 & 23 & 11,4 \\
\hline Obesidade grau II & 4 & 1,8 & 9 & 4,5 \\
\hline Total & 220 & 100,0 & 201 & 100,0 \\
\hline \multicolumn{5}{|c|}{ Circunferência da cintura $(\mathrm{cm})$} \\
\hline Menor risco & 84 & 38,18 & - & - \\
\hline Risco aumentado & 82 & 37,27 & - & - \\
\hline Alto risco & 54 & 24,55 & - & - \\
\hline Total & 220 & 100,0 & - & - \\
\hline
\end{tabular}

Tabela 2. Valores médios e medianos de medidas antropométricas, antes e após o diagnóstico, de portadores de câncer de próstata. Fortaleza (CE), 2006.

\begin{tabular}{|c|c|c|c|c|c|c|c|}
\hline \multirow{2}{*}{ Variável } & \multicolumn{3}{|c|}{ Anterior à doença } & \multicolumn{3}{|c|}{ Atual } & \multirow{2}{*}{ Valor- $p$} \\
\hline & Média & Mediana & DP & Média & Mediana & DP & \\
\hline Peso & 68,24 & 68,00 & 11,06 & 67,77 & 67,00 & 10,95 & 0,949 \\
\hline Altura§ & - & - & - & 162,21 & 162,55 & 5,99 & - \\
\hline$I M C^{*}$ & 25,93 & 25,70 & 4,04 & 25,80 & 25,41 & 3,91 & 0,710 \\
\hline $\mathrm{CC}^{\S}$ & - & - & - & 96,37 & 96,45 & 9,56 & - \\
\hline
\end{tabular}

${ }^{*} \mathrm{n}=201 ;{ }^{\boldsymbol{s}_{n}=220 ;}{ }^{\mathbf{f}}$ Teste dos Postos Sinalizados de Wilcoxon.

DP: desvio-padrão; IMC: índice de massa corporal; CC: circunferência da cintura.

$54,2 \%$ dos 201 homens que souberam informar o peso anterior, tendo sido a média desse IMC no grupo de $25,9 \mathrm{~kg} / \mathrm{m}^{2}\left(18,0-37,5 \mathrm{~kg} / \mathrm{m}^{2}\right)$.

Quando os portadores de câncer de próstata foram investigados quanto à distribuição de gordura corporal, pela medida de circunferência da cintura (CC), a maioria deles $(61,8 \%)$ apresentou um risco aumentado ou alto risco para desenvolver doenças crônicas. A CC média do grupo foi de $96,4 \mathrm{~cm}$, variando de 68,3 a $128,6 \mathrm{~cm}$.

\section{S C U S S Ã O}

A média de idade encontrada para o grupo de portadores de câncer de próstata estudado foi superior (72,88 anos, variando de 52 a 97 anos) à 
encontrada pelos estudos de Maclnnis et al. ${ }^{6}, 61,2$ anos, Eichholzer et al. .7 63,8 anos, Schuurman et al. ${ }^{12}, 63,8$ anos e de Presti Jr. et al. ${ }^{13}, 67$ anos (62-72 anos), sugerindo ser o grupo do presente estudo ainda mais idoso que a maioria dos grupos de portadores da afecção. Apenas o estudo de Hsing et al. ${ }^{14}$ detectou uma média de idade semeIhante à dos pacientes cearenses, 73 anos (50-94 anos). Entretanto, mesmo com esses achados, não se pode afirmar que o câncer de próstata está atingindo homens de idade mais avançada no estado do Ceará que em outras localidades, pois tais dados podem ser atribuídos, em parte, ao diagnóstico tardio que ocorre entre os cearenses. O diagnóstico do câncer, de maneira geral, é feito mais precocemente nos países desenvolvidos que naqueles em desenvolvimento, assim também como o acesso aos exames diagnósticos ocorre mais tardiamente nos estados das regiões menos desenvolvidas (Norte e Nordeste) que nas demais regiões do Brasil ${ }^{15}$.

O grupo apresentou-se com uma média de peso corporal bastante inferior $(67,6 \mathrm{~kg})$ às dos estudos com pacientes australianos ${ }^{6}, 80,5 \mathrm{~kg}$ e dos países baixos ${ }^{12}, 77,8 \mathrm{~kg}$. Menos da metade (44,6\%) dos portadores de câncer de próstata avaliados por Andersson et al. ${ }^{8}$ pesavam até $75,0 \mathrm{~kg}$, enquanto no presente estudo, esse percentual foi bem mais alto $(75,8 \%)$. Entretanto, tais achados podem estar associados à estatura mais elevada entre os homens dos estudos internacionais, já que é conhecido que a renda e as condições de vida influenciam o desenvolvimento e, conseqüentemente, a estatura que o indivíduo atingirá na fase adulta ${ }^{16}$. Dados da Pesquisa Nacional sobre Saúde e Nutrição, realizada em 1989, confirmam o fato de que, mesmo no segmento da população de melhores condições de renda, a mediana da estatura dos adultos brasileiros não alcança a estatura de populações de primeiro mundo ${ }^{17}$. Já quando comparados aos pacientes de países orientais, reconhecidamente como sendo de menor tamanho corporal (peso e altura), em sua maioria ${ }^{14}$, os cearenses apresentaram-se mais pesados, tanto considerando o peso atual, aferido no momento da entrevista $(67,64$ vs 60,4kg), como o peso anterior à doença e ao tratamento $(68,24$ versus $61,3 \mathrm{~kg}$ ). Em ambos os trabalhos houve um sutil aumento do peso corporal dos homens com o passar do tempo, embora sem resultados significantes (Tabela 2). Confrontando com resultados de estudos nacionais, constatou-se um peso superior no grupo aqui avaliado, quando comparado ao peso médio encontrado por Navarro et al. ${ }^{18}$ em pacientes com diagnóstico de neoplasias, entre outras doenças, do Hospital das Clínicas de Ribeirão Preto (SP), com idade média de 51 anos, que foi de $61 \mathrm{~kg}$.

Diante da associação entre renda e condições de vida e a estatura na fase adulta, já era esperada uma estatura média inferior entre os cearenses $(162,2 \mathrm{~cm})$, quando comparados a pacientes de países desenvolvidos: $171,4 \mathrm{~cm}^{6} \mathrm{e}$ $176,3 \mathrm{~cm}^{13}$. Enquanto no estudo de Andersson et al. ${ }^{8}$, apenas $33,5 \%$ da amostra apresentou altura inferior a $172 \mathrm{~cm}$, no presente estudo, quase toda a amostra $(95,4 \%)$ encontrava-se nessa faixa de estatura. Ao contrário do que ocorreu com o peso corporal, quando comparados aos chineses (altura média $=167,8 \mathrm{~cm})^{14}$, os cearenses apareceram com uma altura menor. Mesmo considerando a média de altura da população brasileira adulta $(168,99 \mathrm{~cm})$ e, especificamente, dos nordestinos adultos $(167,06 \mathrm{~cm})$, de acordo com dados da Pesquisa de Orçamentos Familiares (POF 2002 - 2003), analisados por Nogueról et al. ${ }^{16}$, os homens estudados mostraram-se mais baixos. Também o estudo de Navarro et al. ${ }^{18}$ com homens hospitalizados com diagnóstico de neoplasias, entre outras afecções, na cidade de Ribeirão Preto, encontrou uma estatura média mais elevada $(168 \mathrm{~cm})$. Desta forma, a estatura possivelmente não constitui fator de risco para a neoplasia prostática entre os cearenses, uma vez que a literatura refere que a altura está positivamente associada ao risco de desenvolver câncer de próstata e, ainda mais fortemente, ao risco de morrer por tal neoplasia ${ }^{8,19}$.

No que tange ao IMC, foram adotados os pontos de corte da $\mathrm{WHO}^{11}$, embora alguns reco- 
mendem pontos de corte diferentes para idosos, sugerindo que o IMC se eleva com a idade ${ }^{20}$. Mas a escolha do parâmetro foi proposital para viabilizar comparações, inclusive com dados recentes nacionais ${ }^{21}$. Com exceção dos chineses ${ }^{14}$ e da população dos países baixos ${ }^{12}$, que apresentaram um IMC atual médio de $21,9 \mathrm{~kg} / \mathrm{m}^{2}$ e $25,0 \mathrm{~kg} / \mathrm{m}^{2}$, respectivamente, os estudos internacionais com portadores de câncer de próstata encontraram valores superiores aos do presente estudo $(25,7 \mathrm{~kg} /$ $\left.\mathrm{m}^{2}\right): 27,4^{6} \mathrm{~kg} / \mathrm{m}^{2}, 27,5^{13} \mathrm{~kg} / \mathrm{m}^{2}$ e $26,3^{7} \mathrm{~kg} / \mathrm{m}^{2}$. Esses resultados concordam com os dados da World Health Organization/Food and Agriculture Organization', que afirmam ser o excesso de peso um problema mais prevalente nos países desenvolvidos e ocidentais, embora esteja em contínuo crescimento nos países em desenvolvimento. Todavia, merece destaque o fato de que, embora o percentual de excesso de peso entre portadores de câncer de próstata encontrado por Giovannucci et al. ${ }^{9}$ tenha sido semelhante $(52,5 \%)$ e, por Bradbury et $a l .{ }^{10}$, até maior $(59,4 \%)$ que o do presente estudo $(53,6 \%)$, os cearenses apresentaram uma proporção de obesidade bem mais elevada (15,0\%) que a dos estudos citados: 7,3\% e $8,3 \%$, respectivamente.

Em confronto com dados nacionais, embora para a população geral e, não especificamente, para portadores da neoplasia prostática, já que dados sobre excesso de peso nestes pacientes não foram encontrados na literatura pesquisada, o elevado percentual de obesidade entre os acometidos com a doença ganha ainda mais destaque. Os resultados da Pesquisa Nacional sobre Saúde e Nutrição (PNSN), realizada pelo Instituto Brasileiro de Geografia e Estatística (IBGE), em 1989, revelaram a presença de excesso de peso em $30,4 \%$ da população brasileira idosa (idade $\geq 60$ anos), sendo 5,7\% obesidade, e, especificamente para a Região Nordeste, esses valores foram, respectivamente, $22,9 \%$ e 3,1 $\%^{21}$, bem inferiores aos dos pacientes do presente estudo. Entretanto, este estudo foi realizado há bastante tempo e, considerando a transição nutricional que vem ocorrendo no Brasil22, seus resultados podem estar subestimados.
Estudos mais recentes mostram um aumento da prevalência de excesso de peso e obesidade, mas, mesmo assim, em nenhum deles foi encontrada uma proporção de obesos tão alta como a do atual estudo. Comparando com os achados para a população brasileira adulta do sexo masculino, de acordo com dados da Pesquisa de Orçamentos Familiares (POF 2002-2003), a proporção de excesso de peso e obesidade entre os portadores de câncer de próstata estudados apresentou-se bem mais elevada: $53,6 \%$ versus $41,0 \%$ (excesso de peso) e 15,0\% vs 8,8\% (obesidade). Também o inquérito domiciliar sobre comportamentos de risco e morbidade referida de doenças e agravos não transmissíveis, realizado em 15 capitais brasileiras e no Distrito Federal, em 2002/2003, pelo Ministério da Saúde (MS), encontrou uma prevalência de excesso de peso de $44,8 \%$, sendo $11,2 \%$ de obesidade entre homens da capital cearense com idade igual ou superior a 15 anos. Para a população com 50 ou mais anos de idade, de ambos os sexos, houve uma prevalência de $56,6 \%$ de excesso de peso (obesidade e sobrepeso) ${ }^{23}$. A Pesquisa sobre Padrões de Vida (PPV), realizada nas Regiões Nordeste e Sudeste pelo IBGE em 1997, encontrou uma prevalência de $36,7 \%$, sendo $6,7 \%$ de obesidade na população adulta dessas regiões ${ }^{24}$. Ainda segundo dados da mesma pesquisa, especificamente entre os idosos do sexo masculino, o excesso de peso esteve presente em 37,5\% e a obesidade em 5,6\% ${ }^{22}$, valores bastante inferiores aos encontrados para o grupo aqui estudado. Estudo realizado por Cabrera \& Jacob Filho ${ }^{24}$ constatou que 9,3\% dos idosos atendidos em serviços ambulatoriais de Londrina eram obesos, e o IMC do grupo foi de $24,9 \mathrm{~kg} / \mathrm{m}^{2}$. Navarro et al. ${ }^{18}$, estudando pacientes do Hospital das Clínicas de Ribeirão Preto (SP), também encontraram uma média de IMC inferior $\left(22 \mathrm{~kg} / \mathrm{m}^{2}\right)$ à do grupo de portadores da neoplasia em questão, embora esses autores tenham incluído portadores de outras afecções no grupo.

Ainda considerando a Pesquisa de Orçamentos Familiares², realizada em 2002-2003, quando comparada aos dados específicos da 
Região Nordeste e do estado do Ceará, conhecidamente com uma menor prevalência de obesidade que os estados e regiões mais ricas do País, a elevada proporção dos pacientes obesos e com sobrepeso estudados reveste-se de mais destaque. Na Região Nordeste, a prevalência de excesso de peso e obesidade entre os homens adultos foi de $32,9 \%$ e $6,7 \%$, respectivamente. No Ceará, esses valores foram de $35,1 \%$ e $7,9 \%$, respectivamente.

A distribuição de gordura corporal, estimada pela circunferência da cintura, foi considerada de risco na maior parte do grupo, que apresentou uma média da medida bem superior $(96,4 \mathrm{~cm})$ à do grupo de portadores de câncer de próstata da China, de $82,1 \mathrm{~cm}$. Entretanto, é conhecido que os orientais apresentam-se, em sua maioria, com medidas antropométricas menores ${ }^{14}$. Desta forma, comparando o grupo cearense com um grupo australiano (origem ocidental), de homens acometidos com a neoplasia prostática, a diferença entre as médias das medidas de CC foi bem menor $(96,4$ vs $94,9 \mathrm{~cm})$, embora sendo ainda levemente superior à medida entre os cearenses.

Estudos nacionais isolados também encontraram médias do indicador inferiores à do presente estudo. Navarro et al. ${ }^{18}$, que estudaram pacientes hospitalizados com diagnóstico de várias doenças, entre elas o câncer, encontraram, entre os homens, uma média de CC de $86 \mathrm{~cm}$, e Ferreira et al. ${ }^{25}$, estudando doadores de sangue da cidade de Cuiabá (MG), encontraram valor de $85,7 \mathrm{~cm}$. Apenas no estudo de Cabrera \& Jacob Filho ${ }^{24}$, que avaliaram pacientes idosos atendidos em serviços ambulatoriais da cidade de Londrina, se observou um valor mais elevado $(93,8 \mathrm{~cm})$ que os dois anteriores, embora ainda menor que o da presente pesquisa.

Ao analisar a evolução de peso corporal e do estado nutricional, com base no IMC, ocorrida entre o período anterior à descoberta da doença e ao início do tratamento até o dia da entrevista do paciente e da aferição de suas medidas antropométricas, não foram observadas alterações significantes. Estes resultados podem ser explicados pelo fato de o câncer de próstata ser uma das neoplasias que causam menos alterações nutricionais ${ }^{26}$.

Embora muitos tipos de câncer sejam considerados doenças de grande poder consumptivo, que têm como uma de suas principais implicações nutricionais a caquexia, caracterizada por anorexia, perda de peso, tanto de tecido adiposo, como muscular e anemia, a desnutrição conseqüente da doença encontra-se mais freqüentemente associada a casos de neoplasia maligna do pulmão, do pâncreas, do rins e do trato gastrintestinal27. O IBRANUTRI, estudo multicêntrico realizado com pacientes internados em hospitais da rede do Sistema Único de Saúde do Brasil (SUS) de grandes cidades brasileiras, verificou a presença de desnutrição em $66,4 \%$ dos pacientes com câncer e, especificamente, no estado do Ceará, essa proporção foi de $72,9 \%$. Nas neoplasias referentes ao trato geniturinário, em que se inclui a da próstata, o estudo encontrou uma prevalência de desnutrição de $54,5 \%$ 27. Assim, observa-se que, ainda que ocorra em proporções mais reduzidas que em outros sítios, a desnutrição associada ao câncer de próstata deve ser considerada. Por outro lado, no presente estudo, mesmo considerando a perda ponderal relatada, esta não se traduziu em colocar o indivíduo como desnutrido na classificação pelo IMC. Na verdade, em termos nutricionais, a preocupação que emerge no grupo avaliado é com o risco representado pelo excesso ponderal, uma vez que a American Cancer Society destaca que tal situação pode afetar adversamente o prognóstico de sobreviventes de câncer ${ }^{28}$.

\section{CONCLUS Ã O}

Os portadores de câncer de próstata estudados apresentaram alta proporção, passada e atual, de excesso de peso e obesidade. Este fato, associado à presença de gordura abdominal verificada no grupo, representou risco para o desenvolvimento de doenças crônicas em geral. Portanto, tal perfil pode ter se constituído, ao longo do 
tempo, em risco para o surgimento da doença em questão. Não se pode, no entanto, deixar de mencionar como limitação metodológica a inexistência de um grupo de comparação englobando homens cearenses não portadores da neoplasia prostática ou qualquer outro tipo de neoplasia, com idade semelhante à do grupo de casos. Mesmo assim, devido à escassez de estudos nacionais e à inexistência, no âmbito local, de estudos que tratem do perfil antropométrico de portadores de câncer de próstata, torna-se relevante a realização de estudos dessa natureza.

Frente à intenção de contribuir para o avanço do conhecimento nesta área, foi possível conhecer o perfil antropométrico de homens cearenses portadores de câncer de próstata, sugerindo-se a implantação de medidas educativas com vistas à prevenção e ao tratamento do excesso de peso e da obesidade. Instituições de referência, como o local do presente estudo, são espaços importantes que devem incluir a avaliação antropométrica como rotina de ações de prevenção, o que viabilizará o encaminhamento de pacientes em situação nutricional indesejável para intervenções específicas antes que tal situação nutricional comece a aumentar o risco de desenvolvimento de doenças. Logicamente, estudos adicionais, com ampliação da amostra e avaliação de outros fatores associados à doença, se fazem necessários para um melhor conhecimento da realidade local no tocante às inter-relações nutrição-câncer de próstata.

\section{A GRADECIMENTOS}

Aos urologistas do Setor de Uro-Oncologia do Instituto do Câncer do Ceará e, em especial, ao chefe do Serviço, Dr. Lúcio Flávio Gonzaga Silva.

\section{COLABORADORES}

S.P. MACHADO responsável pela estruturação do manuscrito, tendo participado de todas as etapas do processo, desde o desenho, a concepção, a análise e a interpretação dos dados. A.C. SAMPAIO participou das etapas de concepção e desenho, além da interpretação dos dados. J.W.O. LIMA contribuiu com o desenho do trabalho e, especialmente, com a análise dos dados.

\section{REFERÊ NCIAS}

1. World Health Organization. Expert Consultation on diet, nutrition and the prevention of chronic diseases. Geneva: WHO; 2003. WHO Technical Report Series, 916.

2. Instituto Brasileiro de Geografia e Estatística. Pesquisa de orçamentos familiares 2002-2003: análise da disponibilidade domiciliar de alimentos e do estado nutricional no Brasil. Rio de Janeiro: IBGE; 2004.

3. World Health Organization. Global strategy on diet, physical activity and health: obesity and overweight. Geneva: WHO; 2003.

4. Donaldson MS. Nutrition and cancer: a review of the evidence for an anti-cancer diet. Nutr J. 2004; 3:19.

5. Oh SW, Yoon YS, Shin SA. Effects of excess weight on cancer incidences depending on cancer sites and histologic findings among men: Korea National Health Insurance Corporation Study. J Clin Oncol. 2005; 23(21):4742-54.

6. Macinnis RJ, English DR, Gertig DM, Hopper JL, Giles GG. Body size and composition and prostate cancer risk. Cancer Epidemiol Biomarkers Prev. 2003; 12(2):1417-21.

7. Eichholzer M, Bernasconi F, Jordan P, Stähelin HB. Body mass index and the risk of male cancer mortality of various sites:17-year follow-up of the Basel cohort study. Swiss Med Wkly. 2005; 135(1-2):27-33

8. Andersson SO, Wolk A, Bergström R, Adami HO, Engholm $\mathrm{G}$, Englund $\mathrm{A}$, et al. Body size and prostate cancer: a 20-year follow-up study among 135006 swedish construction workers. J Nat Cancer Inst. 1997; 89(5):385-9.

9. Giovannucci E, Rimm EB, Liu Y, Leitzmann $M$, Stampfer MJ, Willett WC. Body mass index and risk of prostate cancer in U.S. health professionals. J Nat Cancer Inst. 2003; 95(16):1240-4.

10. Bradbury BD, Wilk JB, Kaye JA. Obesity and the risk of prostate cancer (United States). Cancer Causes Control. 2005; 16(6):637-41.

11. World Health Organization. Obesity: preventing and managing the global epidemic. Geneva: WHO; 1998. Report of a WHO consultation on obesity. 
12. Schuurman AG, Goldbohm RA, Dorant E, van Den Brant PA. Anthropometry in relation to prostate cancer risk in the Netherlands cohort study. Am J Epidemiol. 2000; 151(6):541-9.

13. Presti JR. JC, Lee U, Brooks JD, Terris MK. Lower body mass index is associated with a higher prostate cancer detection rate and less favorable pathological features in a biopsy population. J Urol. 2004; 171(6):2199-202.

14. Hsing AW, Deng J, Sesterhenn IA, Mostofi FK, Stanczyk FZ, Benichou J, et al. Body size and prostate cancer: a population-based case-control study in China. Cancer Epidemiol Biomarkers Prev. 2000; 9(12):1335-41.

15. Arregi MMU. Registro hospitalar de câncer: cinco anos de experiência no Instituto do Câncer do Ceará, Brasil. Rev Bras Cancerol. 2000; 46(4): 377-87.

16. Nogueról LP, Shikida CN, Monasterio LM. Seis centímetros: uma análise antropométrica da POF 2002-2003. Anais do $7^{\circ}$ Encontro de Economia da Região Sul-ANPEC SUL; 2005.

17. Anjos LA. Índice de massa corporal como indicador do estado nutricional de adultos: revisão da literatura. Rev Saúde Pública. 1992; 26(6):431-6.

18. Navarro AM, Stedille MS, Unamuno MRDL, Marchini JS. Distribuição de gordura corporal em pacientes com e sem doenças crônicas: uso da relação cintura-quadril e do índice de gordura do braço. Rev Nutr. 2001; 14(1):37-41.

19. Engeland A, Tretli S, Bjorge T. Height, body mass index and prostate cancer: a follow-up of 950000 Norwegian men. Br J Cancer. 2003; 89(7):1237-42.

20. Santos DM, Sichieri R. Body mass index and measures of adiposity among elderly adults. Rev Saúde Pública. 2005; 39(2):163-8.

21. Tavares EL, Anjos LA. Perfil antropométrico da população idosa brasileira. Resultados da Pesquisa Nacional sobre Saúde e Nutrição. Cad Saúde Pública. 1999; 15(4):759-68.
22. Brasil. Ministério da Saúde. Secretaria de Vigilância em Saúde. Inquérito domiciliar sobre comportamentos de risco e morbidade referida de doenças e agravos não transmissíveis: Brasil, 15 capitais e Distrito Federal, 2002-2003. Rio de Janeiro: Ministério da Saúde; 2004.

23. Campos MAG, Pedroso ERP, Lamounier JA, Colosimo EA, Abrantes MM. Estado nutricional e fatores associados em idosos. Rev Assoc Med Bras. 2006; 52(4):214-21.

24. Cabrera MAS, Jacob Filho W. Obesidade em idosos: prevalência, distribuição e associação com hábitos e co-morbidades. Arq Bras Endocrinol Metab. 2001; 45(5):494-501.

25. Ferreira MG, Valente JG, Gonçalves-Silva RMV, Sichieri R. Acurácia da circunferência da cintura e da relação cintura-quadril como preditores de dislipidemias em estudo transversal de doadores de sangue de Cuiabá, Mato Grosso, Brasil. Cad Saúde Pública. 2006; 22(2):307-14.

26. Waitzberg DL, Alves CC, Torrinhas RSMM. Incidência da desnutrição em câncer. In: Waitzberg DL. Dieta, nutrição e câncer. São Paulo: Atheneu; 2004. 32:269-78.

27. Waitzberg DL, de Nardi L, Ravacci GR, Torrinhas RSMM. Síndrome da anorexia e caquexia em câncer: abordagem terapêutica. In: Waitzberg DL. Dieta, nutrição e câncer. São Paulo: Atheneu; 2004. 38: 334-52.

28. American Cancer Society. Nutrition and physical activity guidelines for cancer prevention: summary. [cited 2008 Nov 30]. Available from: <http://www. cancer/org/docroot/PED/content/PED_3_2X_Diet_ and_Activity_Factors_That_Affect_Risks.asp? sitearea $=\mathrm{MH}>$.

Recebido em: 24/4/2007

Versão final reapresentada em: 7/1/2009 Aprovado em: 9/3/2009 